\title{
Optimization of the Turbulence Model on Numerical Simulations of Flow Field within a Hydrocyclone
}

\author{
Yan Xu, Zunce Wang, Lin Ke, Sen Li, and Jinglong Zhang \\ School of Mechanical Science and Engineering, Northeast Petroleum University, Developing Road, P.O. Box 199, \\ High-Tech District, Daqing, Heilongjiang 163318, China
}

Correspondence should be addressed to Zunce Wang; wangzc@nepu.edu.cn

Received 25 September 2014; Revised 26 January 2015; Accepted 13 April 2015

Academic Editor: Marc Dahan

Copyright (C) 2015 Yan Xu et al. This is an open access article distributed under the Creative Commons Attribution License, which permits unrestricted use, distribution, and reproduction in any medium, provided the original work is properly cited.

Reynolds Stress Model and Large Eddy Simulation are used to respectively perform numerical simulation for the flow field of a hydrocyclone. The three-dimensional hexahedral computational grids were generated. Turbulence intensity, vorticity, and the velocity distribution of different cross sections were gained. The velocity simulation results were compared with the LDV test results, and the results indicated that Large Eddy Simulation was more close to LDV experimental data. Large Eddy Simulation was a relatively appropriate method for simulation of flow field within a hydrocyclone.

\section{Introduction}

As separation equipment, a hydrocyclone is widely used in petroleum industry, marine industry, and water treatment industry. The fidelity of CFD predictions for turbulent flows is highly dependent upon the quality of the turbulence modelling. This is especially true when it comes to the flow in hydrocyclone, because the salient features include high swirl, three-dimensional boundary layers with strong streamline curvature. The studies of the turbulence model on numerical simulations of a hydrocyclone indict that different degrees of defects exist in mixed length model, normal $k-\varepsilon$ model, and various modified $k-\varepsilon$ model established based on eddy viscosity hypothesis. The basic solution to solve the defects of aforementioned different models lies in giving up turbulence model established on isotropic eddy viscosity hypothesis, while adopting anisotropic Reynolds Stress Model (RSM) and Large Eddy Simulation (LES) instead [1-3].

For the past few years, LES has been applied to the numerical investigation of flow within a hydrocyclone. Delgadillo and Rajamani $[4,5]$ used LES, RSM, and renormalization group $k-\varepsilon$ turbulence model to simulate the flow of a hydrocyclone. By comparison of their results and experimental data, this showed that LES was more accurate. Schmidt et al. [6] adopted LES to process unsteady characteristics of the fluid and gained good results. Souza et al. $[4,7,8]$ used LES to simulate the flow field of a hydrocyclone; the results showed, although LES need more computational cost, they all got very precise velocity distribution, especially for the tangential velocity.

RSM and LES were, respectively, used in this paper to conduct numerical simulations for the three-dimensional flow field of a hydrocyclone, and the simulation results were compared with the experimental data. The distribution of flow field in a hydrocyclone was gained.

\section{RSM Control Equations}

2.1. Basic Governing Equations. For incompressible flow, the Reynolds-averaged Navier-Stokes equations:

$$
\begin{gathered}
\frac{\partial u_{i}}{\partial x_{i}}=0 \\
\frac{\partial \rho u_{i}}{\partial t}+\rho \frac{\partial}{\partial x_{j}}\left(u_{i} u_{j}\right)=-\frac{\partial p}{\partial x_{i}}+\frac{\partial}{\partial x_{j}}\left(\mu \frac{\partial u_{i}}{\partial x_{j}}-\rho \overline{u_{i}^{\prime} u_{j}^{\prime}}\right) .
\end{gathered}
$$

In these equations, $x_{i}(i=1,2,3)$ is coordinate component, $u_{i}, u_{j}(i, j=1,2,3)$ is the time-mean velocity component, $p$ is the time-mean average pressure, $\mu$ is dynamic viscosity of 
the fluid, $\rho$ is the fluid density, and $\overline{u_{i}^{\prime} u_{j}^{\prime}}$ is the Reynolds stress component.

2.2. RSM Turbulence Model. Solve the Reynolds stress equations to get closed equations. One has

$$
\frac{\partial}{\partial t}\left(\rho \overline{u_{i}^{\prime} u_{j}^{\prime}}\right)+\frac{\partial}{\partial x_{k}}\left(\rho u_{k} \overline{u_{i}^{\prime} u_{j}^{\prime}}\right)=D_{i j}+p_{i j}+\varphi_{i j}+\varepsilon_{i j} .
$$

In the equation, every concrete form in the right hand is as follows:

$$
D_{i j}=\frac{\partial}{\partial x_{k}}\left(\frac{\mu_{t}}{\sigma_{k}} \frac{\partial \overline{u_{i}^{\prime} u_{j}^{\prime}}}{\partial x_{k}}+\mu \frac{\partial \overline{u_{i}^{\prime} u_{j}^{\prime}}}{\partial x_{k}}\right),
$$

where $\mu_{t}$ is the turbulent viscosity, $\sigma_{k}=0.82$. Consider

$$
\begin{aligned}
p_{i j} & =-\rho\left(\overline{u_{i}^{\prime} u_{j}^{\prime}} \frac{\partial u_{j}}{\partial x_{k}}+\overline{u_{j}^{\prime} u_{k}^{\prime}} \frac{\partial u_{i}}{\partial x_{k}}\right) \\
\varphi_{i j} & =-C_{1} \rho \frac{\varepsilon}{k}\left(\overline{u_{i}^{\prime} u_{j}^{\prime}}-\frac{2}{3} k \delta_{i j}\right)-C_{2}\left(P_{i j}-\frac{1}{3} P_{k k} \delta_{i j}\right),
\end{aligned}
$$

where $C_{1}=1.8, \delta_{i j}$ is Kronecker delta, $k$ is turbulence energy, $\varepsilon$ is turbulent dissipation rate, and $\varepsilon_{i j}=-(2 / 3) \rho \varepsilon \delta_{i j}$.

Then the closed Reynolds stress equations are got.

\section{LES Control Equations}

3.1. Basic Governing Equations. In LES, the governing equations are obtained by spatially filtering the Navier-Stokes equations. The large turbulent scales are computed explicitly, while the small scales are modelled using Subgrid Scale (SGS) models. The SGS models describe interactions between the resolved and unresolved scales.

For incompressible flow, the governing equations employed for LES are obtained by filtering the timedependent; applying the filtering operation to the continuity and momentum equations gives

$$
\begin{aligned}
& \frac{\partial \rho}{\partial t}+\frac{\partial}{\partial x_{i}}\left(\rho \bar{u}_{i}\right)=0 \\
& \frac{\partial}{\partial t}\left(\rho \bar{u}_{i}\right)+\frac{\partial}{\partial x_{j}}\left(\rho \bar{u}_{i} \bar{u}_{j}\right) \\
& =-\frac{\partial \bar{p}}{\partial x_{i}}+\mu \frac{\partial^{2} \bar{u}_{i}}{\partial x_{j} x_{j}}+\frac{\partial}{\partial x_{j}}\left(\rho\left(\bar{u}_{i} \bar{u}_{j}-\bar{u}_{i} u_{j}\right)\right) .
\end{aligned}
$$

$\bar{\tau}_{i j}=\bar{u}_{i} \bar{u}_{j}-\bar{u}_{i} u_{j}$ is SGS stress and is computed to close the model.

3.2. Subgrid Scale Model. The subgrid scale turbulence models employ the Boussinesq hypothesis as in the RANS models, computing subgrid scale turbulent stresses from

$$
\tau_{i j}-\frac{1}{3} \tau_{k k} \delta_{i j}=-2 \mu_{t} \bar{S}_{i j}
$$

where $\mu_{t}$ is subgrid scale turbulent viscosity and $\bar{S}_{i j}$ is the rateof-strain tensor for the resolved scale defined by:

$$
\bar{S}_{i j} \equiv \frac{1}{2}\left(\frac{\partial \bar{u}_{i}}{\partial x_{j}}+\frac{\partial \bar{u}_{j}}{\partial x_{i}}\right) .
$$

Kinetic Energy Transport Subgrid Scale Model was proposed by Kim and Menon [9], which acquired favorable application on complex flow of nonequilibrium turbulence. This paper adopted the Kinetic Energy Transport model to calculate the complex flow in hydrocyclone. In the Kinetic Energy Transport model, the eddy viscosity is modeled by

$$
\mu_{t}=C_{k} \rho k_{\mathrm{sgs}}^{1 / 2} \Delta_{f},
$$

where Subgrid Scale Kinetic Energy is computed using

$$
k_{\mathrm{sgs}}=\frac{1}{2}\left(\overline{u_{k}^{2}}-\bar{u}_{k}^{2}\right),
$$

where $\Delta_{f}$ is the filter size computed from $\Delta_{f}=V^{1 / 3}$; SGS stress can then be written as

$$
\tau_{i j}-\frac{2}{3} \rho k_{\mathrm{sgs}} \delta_{i j}=-2 C_{k} \rho k_{\mathrm{sgs}}^{1 / 2} \Delta_{f} \bar{S}_{i j} .
$$

$k_{\mathrm{sgs}}$ is obtained by solving its transport equation. Consider

$$
\begin{aligned}
\rho \frac{\partial \bar{k}_{\mathrm{sgs}}}{\partial t}+\rho \frac{\partial \bar{u}_{j} \bar{k}_{\mathrm{sgs}}}{\partial x_{j}}= & -\tau_{i j} \frac{\partial \bar{u}_{i}}{\partial x_{j}}-C_{\varepsilon} \rho \frac{k_{\mathrm{sgs}}^{3 / 2}}{\Delta f} \\
& +\frac{\partial}{\partial x_{j}}\left(\frac{\mu_{t}}{\sigma_{k}} \frac{\partial k_{\mathrm{sgs}}}{\partial x_{j}}\right) .
\end{aligned}
$$

In the above equations, the model constants, $C_{k}$ and $C_{\varepsilon}$, are determined dynamically; $\sigma_{k}$ is hardwired to 1.0.

\section{The Numerical Calculation Model of Flow Field within a Hydrocyclone}

4.1. Calculation Domain and the Grid. Double conical hydrocyclone was selected as the research object in this paper. Its main diameter $D=56 \mathrm{~mm}$. The fluid that flowed into the hydrocyclone through two inlets located at the tangential direction formed strong swirl turbulence in it and then outflowed from two outlets of underflow and overflow. The geometry is shown in Figure 1.

The hexahedral computational grids were generated using the grid generator ICEM CFD, and the pipe center adopts an $O$-block grid. In order to guarantee the solution requirement of LES on the viscous sublayer, the grid height of the first layer is $L=2.0 \times 10^{-5} \mathrm{~m}$, and the grid extends in a radial direction with the scaling factor of 1.08 . The final grid had about 2.0 $\times 10^{6}$ cells. The near wall grid satisfies $y+=O(1)$, which guarantees the accurate seizing of a small scale eddy in the flow field. The grid is shown in Figure 2.

4.2. Boundary Conditions. In this paper, the velocity $u$ of two inlets is $0.5 \mathrm{~m} / \mathrm{s}$, with the hydraulic diameter of $16.6 \mathrm{~mm}$. 


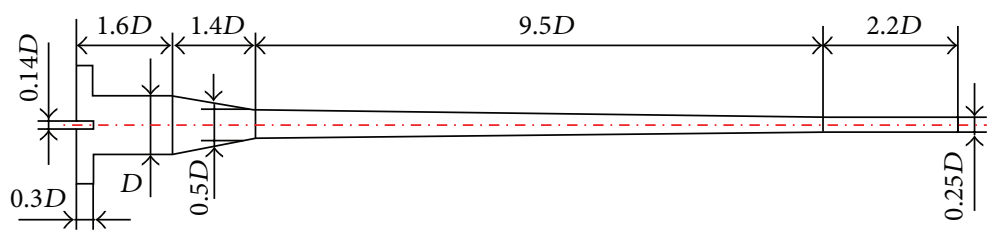

FIGURE 1: Geometry of the double conical hydrocyclone.

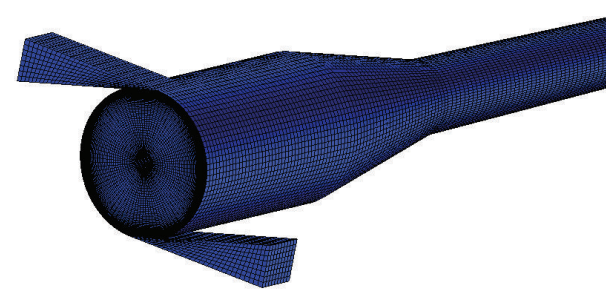

FIGURE 2: Graphics display of grid.
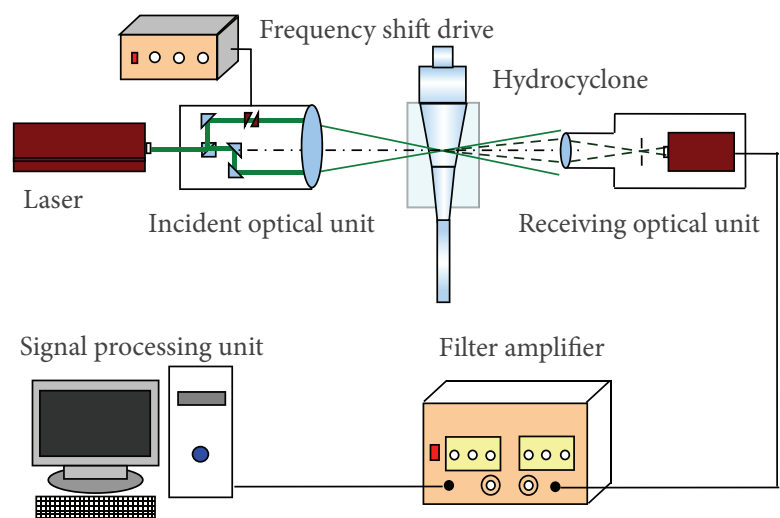

FIGURE 3: Schematic diagram of LDV.

According to $I=0.16(\mathrm{Re})^{-1 / 8}$, the turbulence intensity $I$ is $5.18 \%$. According to the formula, $k=3 / 2(U * I)^{2}$, the turbulence energy $k$ can be obtained as $0.001 \mathrm{~m}^{2} / \mathrm{s}^{2}$. The underflow and overflow are disposed as the full developed $\partial \Phi / \partial z=0$, and the split ratio is $95 \%$ and $5 \%$, respectively. The boundary conditions of wall are set as no slip wall, whose velocity components are $u=0, v=0$, and $w=0$. In the RSM simulation, Nonequilibrium Wall Functions were used. In the LES, Wall Functions were not used.

\subsection{Numerical Calculation Method. ANSYS FLUENT 14.5} was used on a 12 Core CPU $2.67 \mathrm{GHz}$ workstation. Control equations are dispersed in space by finite volume method. Detailed numerical calculation methods of RSM and LES are shown in Table 1. In the LES method, courant number (courant number = time step size/(unit grid length/the velocity in the grid)) is approximately equal to 1 . The time step size is calculated by the courant number. The simulation was run until steady state. After steady state was reached, a real time of one second was simulated with a time step of

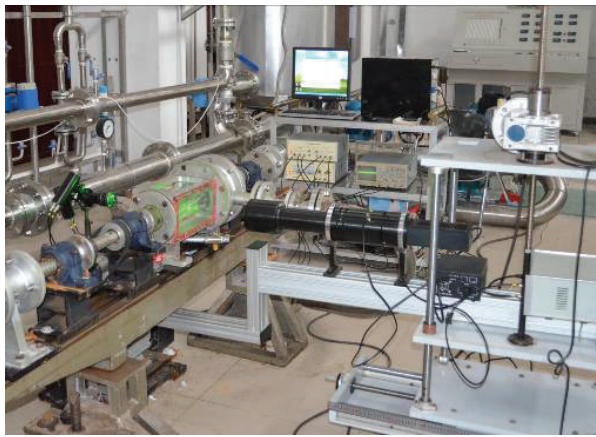

FIGURE 4: Experiment equipment.

TABLE 1: Numerical calculation method of RSM and LES simulations.

\begin{tabular}{lcc}
\hline Analogy method & RSM & LES \\
\hline Grid quantity & $9.0 \times 10^{5}$ & $2.0 \times 10^{6}$ \\
$\begin{array}{l}\text { Pressure-velocity coupling } \\
\text { method }\end{array}$ & SIMPLEC & PISO \\
Gradient interpolation & Least square & Least square \\
& method & method \\
Pressure interpolation & PRESTO! & Standard \\
Convection interpolation & QUICK & Second Order \\
Convergence precision & $1 \times 10^{-5}$ & Upwind \\
\end{tabular}

$0.0001 \mathrm{sec}$. An average of over one thousand time steps was taken to record the velocity profile and other results.

\section{The Flow Field Velocity Test Experiment}

The flow field test apparatus is established in the ratio of $1: 1$ according to the simulated hydrocyclone. SCD-23 twodimensional Laser Doppler Velocimeter (LDV) of Tsinghua University is used to conduct a LDV test on the internal flow field. Speed measuring precision is $\pm 0.5 \%$. This process consists of a supply tank, a power pump, flowmeters, pressure metering devices, a control valve, a hydrocyclone, the LDV, and so forth. A screw pump is used to feed fluid in cycle, and the test fluids use running water. The LDV system and the experimental apparatus are shown in Figures 3 and 4. The inlet velocity is $0.5 \mathrm{~m} / \mathrm{s}$ in experiment process. The values of tangential and axial velocities of three sections with $z=$ $150 \mathrm{~mm}, z=200 \mathrm{~mm}$, and $z=300 \mathrm{~mm}$ in hydrocyclone are 


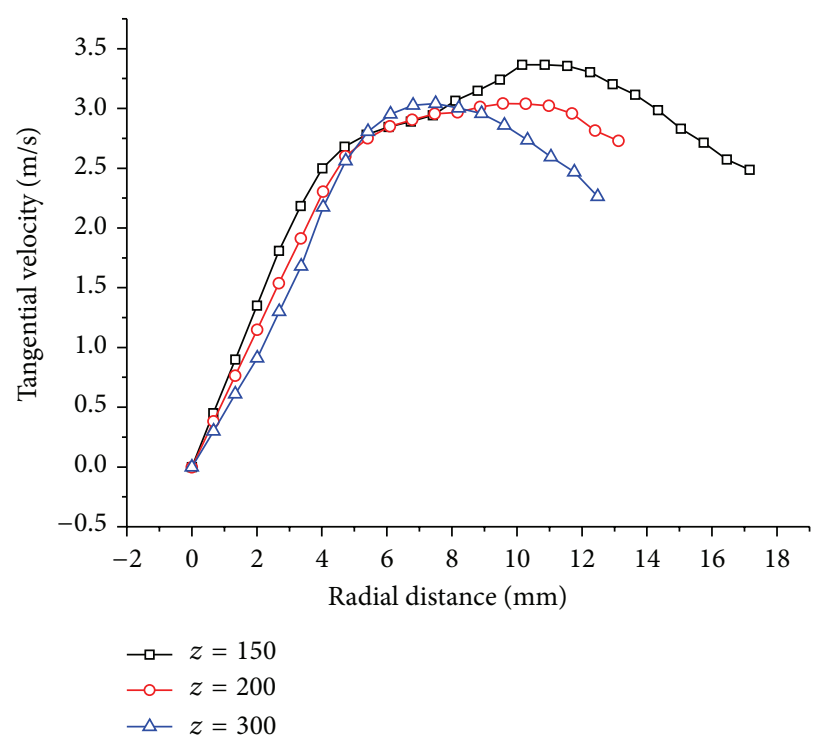

FIgURE 5: Tangential velocity distribution.

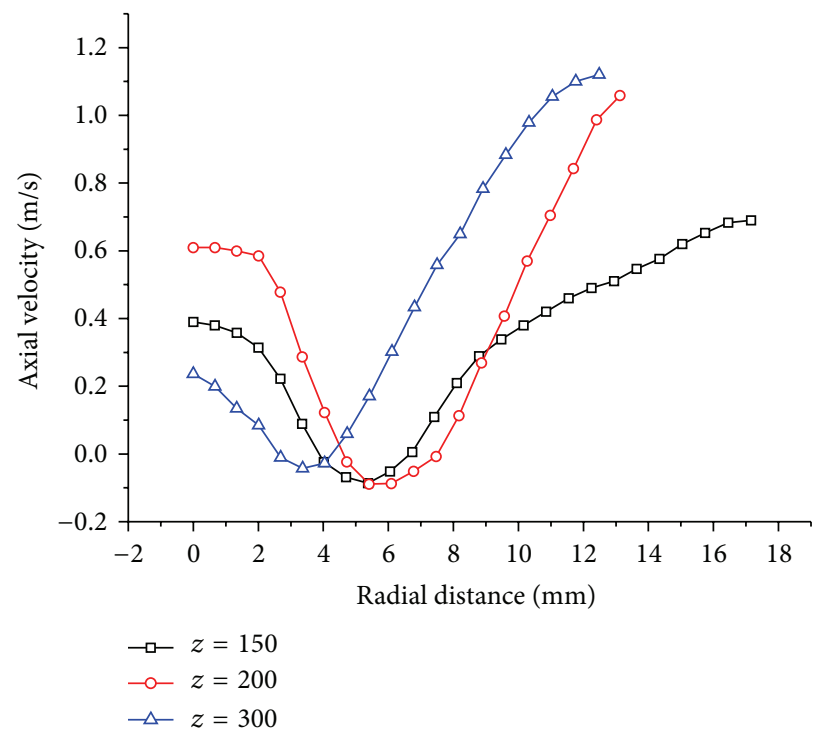

FIgURE 6: Axial velocity distribution.

obtained, and the specific results are shown in Figures 5, 6, and 7.

\section{Analysis of Computing Result}

6.1. Turbulent Kinetic Energy. The turbulent kinetic energy distribution in hydrocyclone was shown in Figure 7. It can be seen that the turbulent kinetic energy was higher near the small conical wall and the overflow in the LES than the RSM simulation, in which there was nearly no higher turbulence kinetic energy generated except from the small cone section. This is because, if we want to get more accurate calculation of turbulent kinetic energy information, the boundary layer flow should be enough computed. As the LES method had enough solutions to its boundary layer, the turbulent kinetic energy generated in the hydrocyclone was predicted relatively accurate. However the RSM method simulated the boundary layer flow by logarithmic ratio which has lost the whole generated information of the turbulent kinetic energy. Therefore, the LES method has more advantages for the prediction of the turbulent kinetic energy of flow field in hydrocyclone than the RSM method.

6.2. The Vorticity Distribution. The vorticity distribution in hydrocyclone was shown in Figure 8. We can see from this figure that there were significant nonzero values in the LES to predict the vorticity near the wall, which appeared to be alternate variation in space and constituted a small scale 


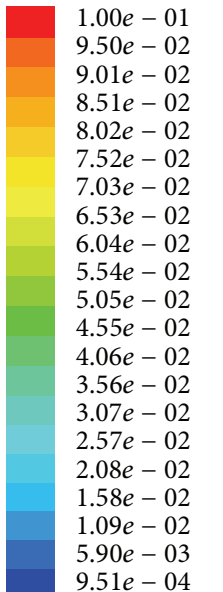

(a) RSM simulation

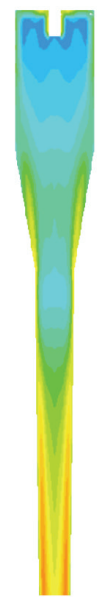

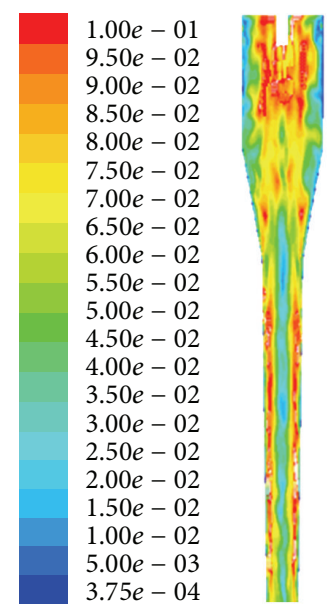

(b) LES

FIGURE 7: Turbulent kinetic energy distribution in hydrocyclone.

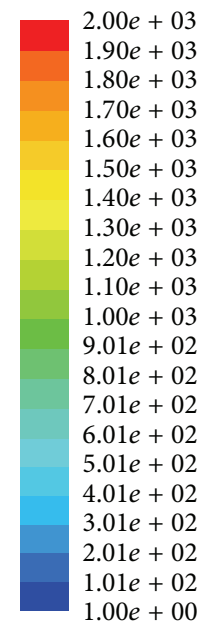

(a) RSM simulation

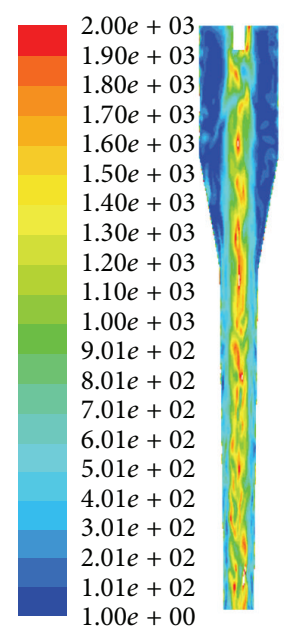

(b) LES

FIGURE 8: The vorticity distribution in hydrocyclone.

vortex structure. With the development of direction of flow to the center, the scale of the vortex increased gradually. Considering the vorticity in the RSM simulation, a lot of small scale vortexes structures were lost. The prediction of central vortexes value was less than the LES results. Thus, the LES can solve more vortex structure of flow field than the RSM and get more turbulent flow pulsation information. It is more advanced in terms of catching small scale vortexes, by the reason that Subgrid Scale Model in the LES can accurately describe the movement of small scale vortex.

\subsection{The Flow Velocity Analysis}

6.3.1. Tangential Velocity. In the velocity field of the hydrocyclone, tangential velocity is predominant, which is an important factor in affecting the separation efficiency. The comparison of RSM simulation results, LES results, and experimental results of the flow field tangential velocity distribution of the various stations in the hydrocyclone can be seen in Figure 9.

As can be seen from the figure, the velocity distribution was made up of forced vortexes and free vortexes, and the axial symmetry of the tangential velocity distribution was better. The RSM simulation results both had large deviations on numerical values and the distribution to the experiment results, and the differences were bigger especially in the free vortex area. The tangential velocity distribution of the LES results was relatively consistent with the experiment results both on the values and the distribution, and the forced vortexes were largely overlapping.

6.3.2. Axial Velocity. In the velocity field of the hydrocyclone, another important speed is the axial velocity. The comparison of RSM simulation results, LES results, and experimental results of the flow field axial velocity distribution of various stations in the hydrocyclone can be seen in Figure 9. In the 


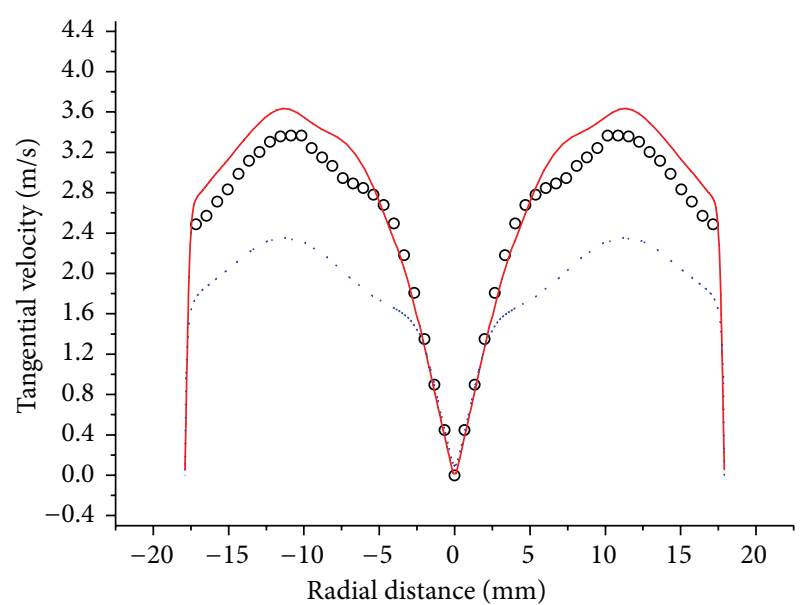

(A1) $z=150 \mathrm{~mm}$ station

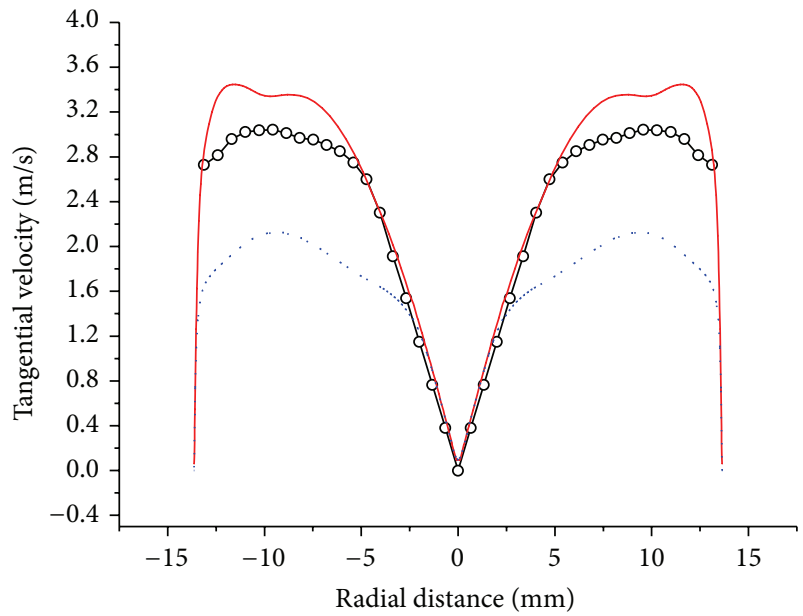

(B1) $z=200 \mathrm{~mm}$ station

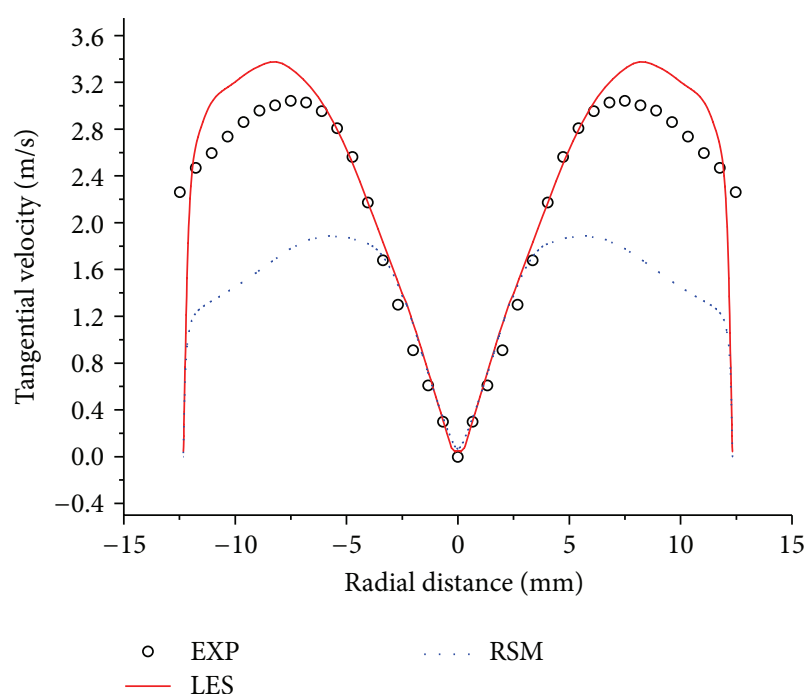

(C1) $z=300 \mathrm{~mm}$ station

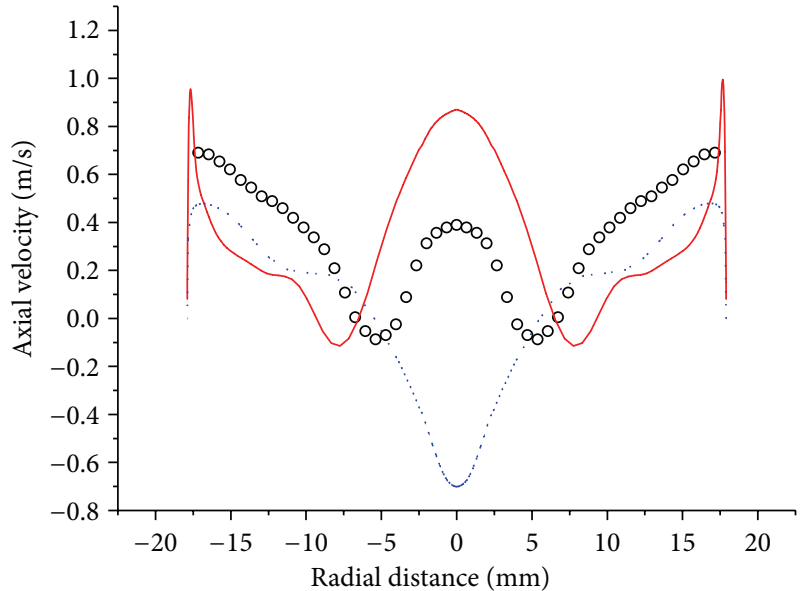

(A2) $z=150 \mathrm{~mm}$ station

(a)

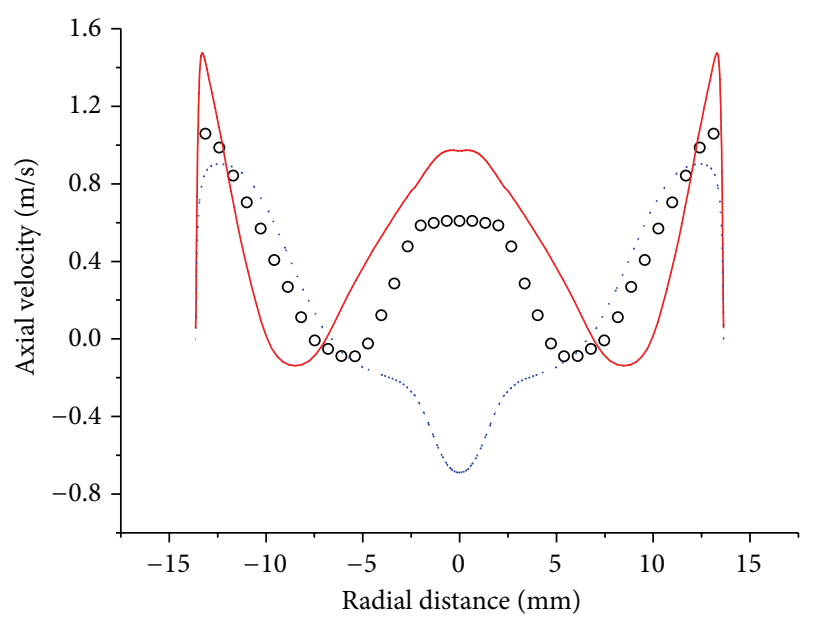

(B2) $z=200 \mathrm{~mm}$ station

(b)

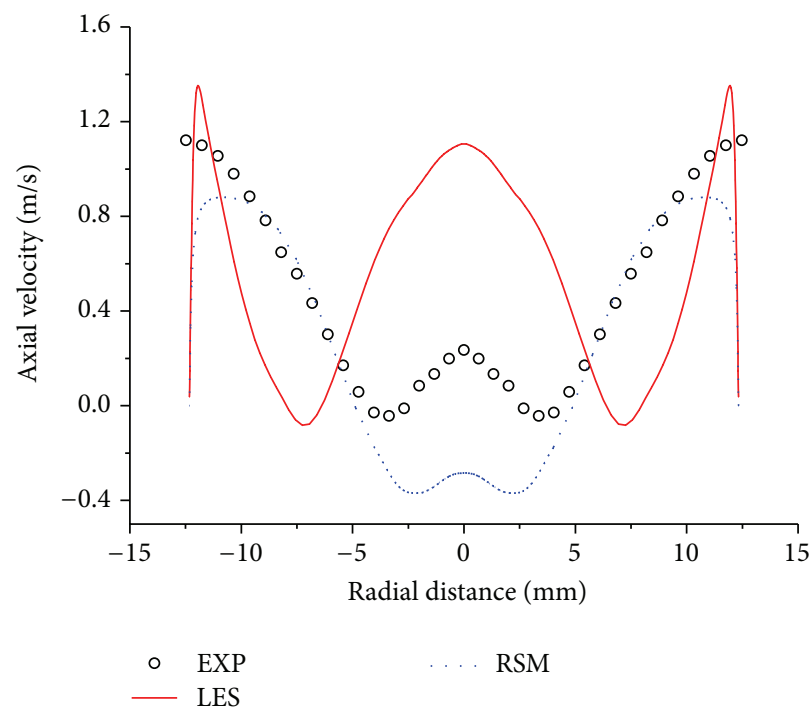

(C2) $z=300 \mathrm{~mm}$ station

(c)

FIGURE 9: Comparison between the tangential and axial velocity at various stations as predicted by the RSM model, the averaged LES results, and the experimental data. 
figure, the positive values denote the fluid outflow from the underflow, while the negative value represented the fluid outflow from the overflow. The axial velocity in the hydrocyclone changed from the wall to the center. The maximum value appeared near the wall, with the direction pointing to the underflow outlets. We can see from the numerical size that the distribution regularity of RSM and LES results agreed well with the measured values, and the RSM was more ideal on numerical values near the wall. Opposite results appeared between the RSM and measured values, while the LES was more close to experimental values near the axis. Considering whole fluctuation rule, the LES method was more close to the LDV measured results.

\section{The Conclusion}

(1) In the calculation of the turbulent kinetic energy and the vorticity of flow field in hydrocyclone, the RSM lost the pulsation characteristics of the small scale vortex structures in spite of solving the boundary layer flow. The LES has the absolute advantages as the Subgrid Scale Model can capture small scale vortex structures more accurately.

(2) The two-dimensional LDV is used to test the flow field of a hydrocyclone, and the velocity distribution of tangential and axial velocities of the various stations in the hydrocyclone under the condition of inlet velocity $0.5 \mathrm{~m} / \mathrm{s}$ has been got.

(3) During the numerical simulation of velocity field in the hydrocyclone, the RSM simulation results both have some deviations on values and the distribution to the experiment results. The LES results agree well with the LDV results. The simulation results of the LES are superior to the RSM.

\section{Conflict of Interests}

There is not any conflict of interests related to this paper.

\section{Acknowledgments}

The research was supported by the National Natural Science Foundation of China (11172061) (11402051), Postdoctoral Fund of Heilongjiang Province of China (LBH-Z12273), and Project in Heilongjiang Province Department of Education of China (ky120444).

\section{References}

[1] K. U. Bhaskar, Y. R. Murthy, M. R. Raju, S. Tiwari, J. K. Srivastava, and N. Ramakrishnan, "CFD simulation and experimental validation studies on hydrocyclone," Minerals Engineering, vol. 20, no. 1, pp. 60-71, 2007.

[2] R. Maddahian, M. Asadi, and B. Farhanieh, "Numerical investigation of the velocity field and separation efficiency of deoiling hydrocyclones," Petroleum Science, vol. 9, no. 4, pp. 511-520, 2012.

[3] J. A. Delgadillo, M. Al Kayed, D. Vo, and A. S. Ramamurthy, "CFD simulations of a hydrocyclone in absence of an air core," Journal of Mining and Metallurgy, Section B: Metallurgy, vol. 48, no. 2, pp. 197-206, 2012.
[4] J. A. Delgadillo and R. K. Rajamani, "A comparative study of three turbulence-closure models for the hydrocyclone problem," International Journal of Mineral Processing, vol. 77, no. 4, pp. 217-230, 2005.

[5] J. A. Delgadillo and R. K. Rajamani, "Exploration of hydrocyclone designs using computational fluid dynamics," International Journal of Mineral Processing, vol. 84, no. 1-4, pp. 252-261, 2007.

[6] S. Schmidt, H. Blackburn M, and M. Rudman, "Impact of outlet boundary conditions on the flow properties within a cyclone," in Proceedings of the 15th Australasian Fluid Mechanics Conference, pp. 13-17, Sydney, Australia, 2004.

[7] F. J. Souza and A. S. Neto, "Preliminary results of large eddy simulations of a hydrocyclone," Revista de Engenharia Térmica, vol. 3, no. 2, pp. 168-173, 2004.

[8] J. Zhang, Z. Wang, and Y. Xu, "Large eddy simulation of flow field in a liquid-liquid hydrocyclone," Chemical Engineering \& Machinery, vol. 40, no. 3, pp. 335-339, 2013.

[9] W. W. Kim and S. Menon, "Application of the localized dynamic subgrid-scale model to turbulent wall-bounded flows, 35th Aerospace Sciences Meeting, Reno, Nev, USA," Tech. Rep., AIAA-97-0210, American Institute of Aeronautics and Astronautics, 1997. 


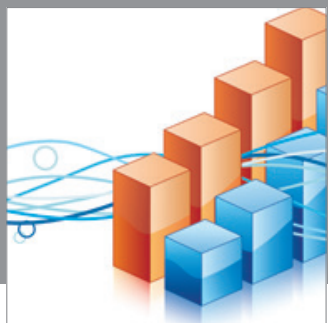

Advances in

Operations Research

mansans

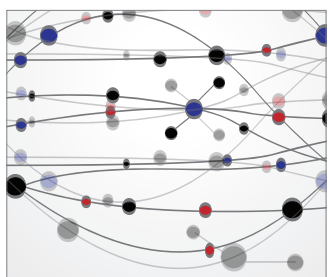

The Scientific World Journal
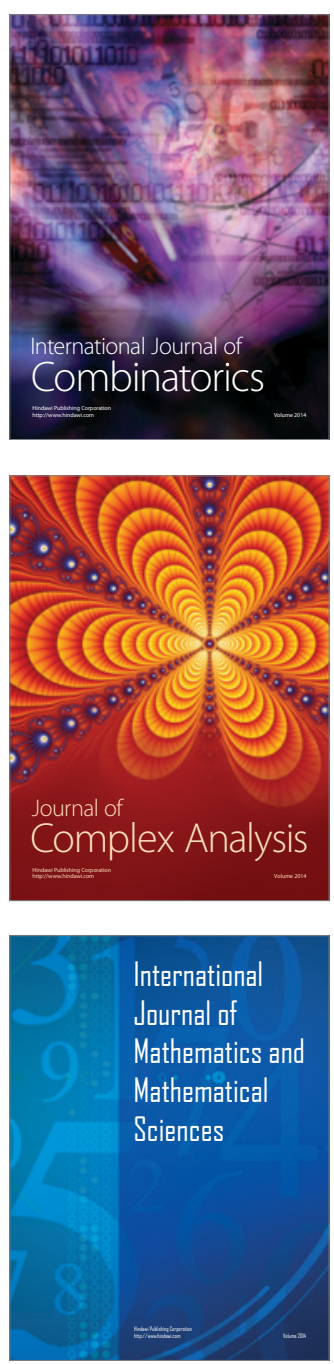
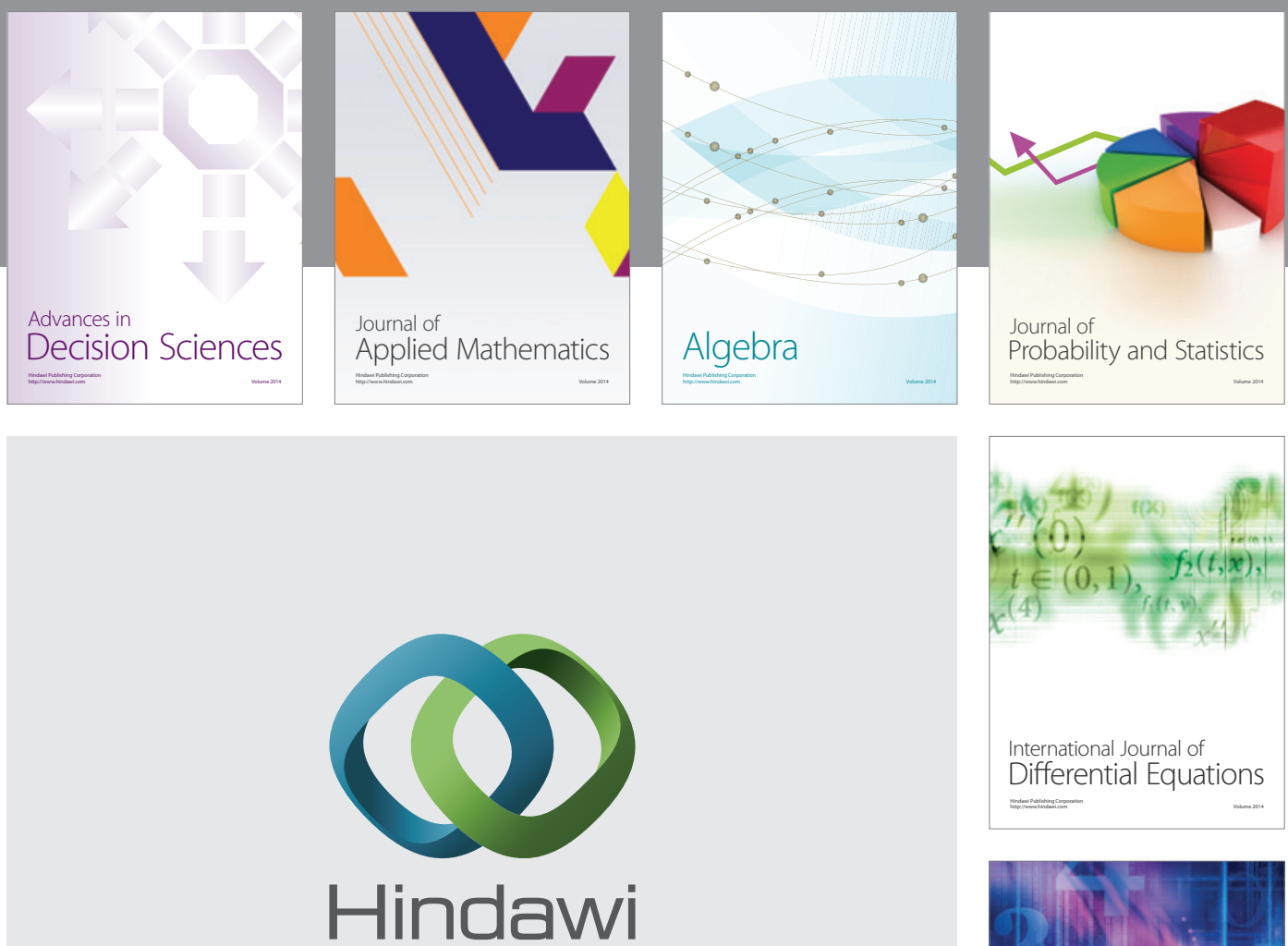

Submit your manuscripts at http://www.hindawi.com
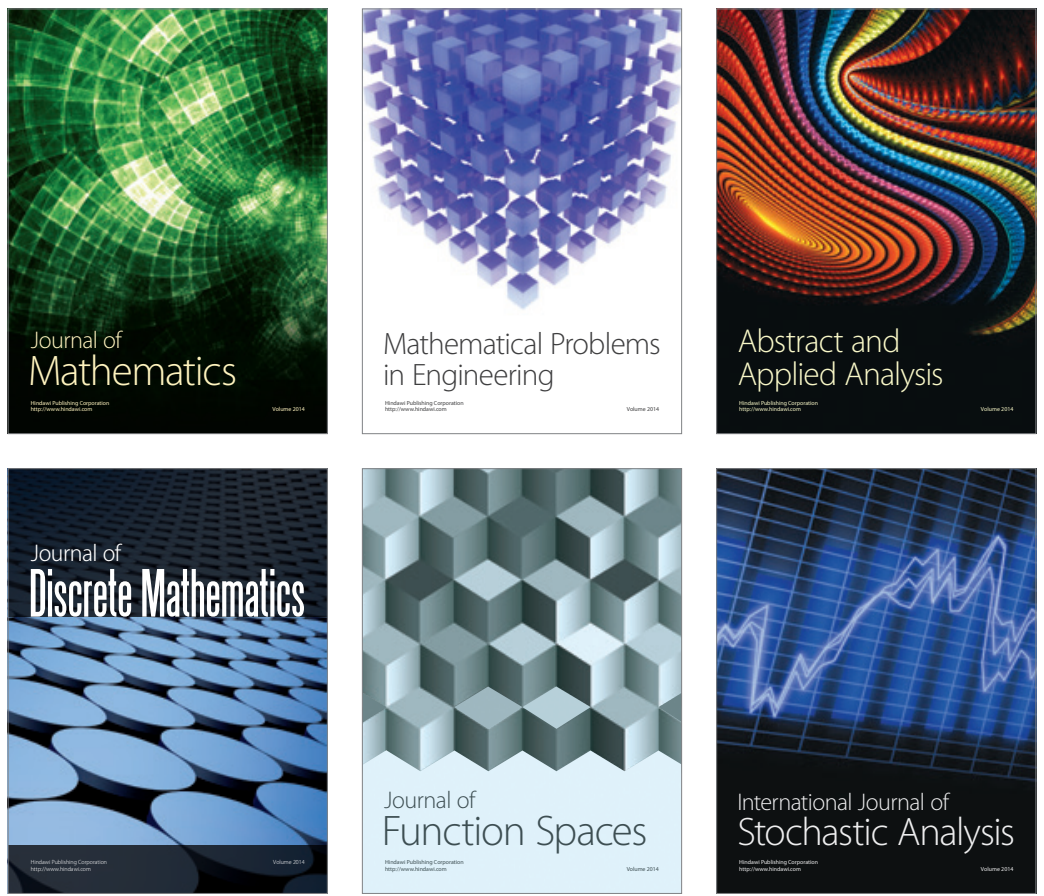

Journal of

Function Spaces

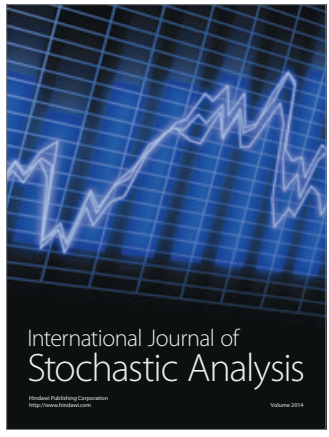

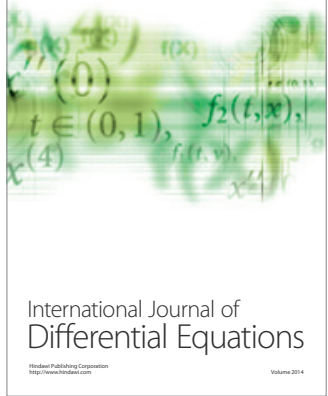
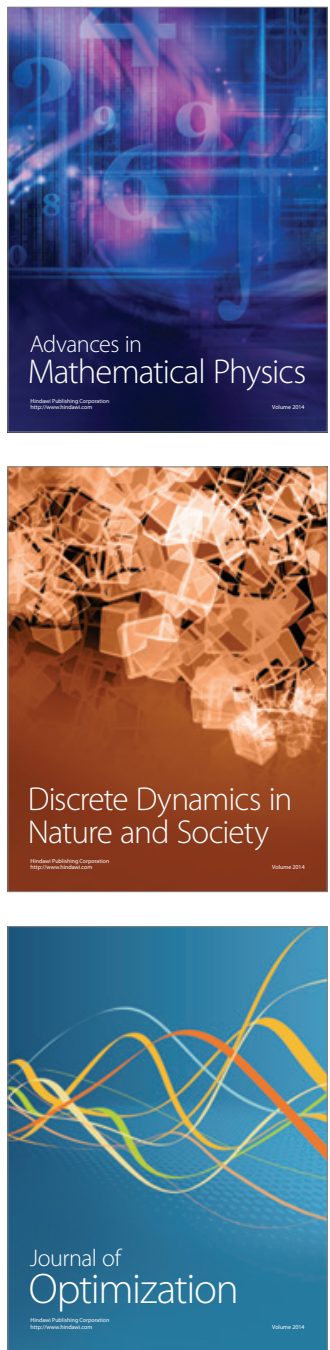